\title{
Avaliação psicológica na aderência terapêutica de tratamento hemodialítico
}

\author{
Psychological assessment in hemodialysis treatment adherence therapy
}

Remerson Russel Martins ${ }^{[0]}$, Joc̃o Carlos Alchier ${ }^{[b]}$

\footnotetext{
${ }^{[a]}$ Mestre em Psicologia pela Universidade Federal do Rio Grande do Norte (UFRN), professor do Departamento de Agrotecnologia e Ciências Sociais da Universidade Federal Rural do Semi-Árido (Ufersa), Mossoró, RN - Brasil, e-mail: remerson@ufersa.edu.br

${ }^{[b]}$ Doutor em Psicologia pela Universidade Federal do Rio Grande do Sul (UFRGS), professor do Departamento de Psicologia da Universidade Federal do Rio Grande do Norte (UFRN), Natal, RN - Brasil, e-mail: jcalchieri@gmail.com
}

Recebido: $11 / 03 / 2011$ Received: 03/11/2011

\section{Resumo}

A aderência terapêutica é conceituada como o grau de concordância entre o comportamento do paciente e as prescrições da equipe de saúde. Avalia-se com o Inventário Millon de Estilos de Personalidade (IMEP) respostas comportamentais da aderência terapêutica ao tratamento hemodialítico. Participantes de um programa de atendimento hemodialítico, 41 mulheres e 39 homens, de 19 a 78 anos ( $\pm 43,4$ anos), de seis meses a 22 anos de hemodiálise $( \pm 4,24$ anos), foram avaliados entre agosto de 2007 e janeiro de 2008, na Grande Natal (RN). Observou-se uma aderência média de $55,97 \%( \pm 18,37 \%)$ e uma associação significativa $(\mathrm{p}<0,05)$ com algumas escalas do IMEP. Assim, os pacientes mais aderentes apresentaram-se mais otimistas, buscando adaptar-se, processando dados objetivos e simbólicos, estabelecendo relacionamentos gregários e flexíveis. Estas características explicaram 37,9\% da variação da pressão arterial pré-diálise. O IMEP discriminou diferentes níveis de aderência, sendo que o uso de diferentes indicadores mostrou-se importante na avaliação da aderência. Os níveis de aderência observados conformam-se ao registrado pela literatura.

Palavras-chave: Avaliação em saúde. Psicometria. Cooperação do paciente. Insuficiência renal crônica.

\section{Abstract}

Compliance is appraised as the agreement level between the patient's behavior and the prescriptions of the health team. We evaluate the Millon Index Personality Styles in the measurement of the compliance with hemodialytic treatment. In this study, 41 women and 39 men, between 19 and 78 years old ( $\pm 43,4$ years), with the range of six months and 22 years of hemodialysis, were evaluated between August of 2007 and January of 2008 in the metropolitan area of Natal (RN). It was observed an average compliance of $55.97 \%$ ( $\pm 18.37 \%$ ) and a significant relation $(p<0.05)$ with some MIPS scales. Thus, the most adherent patients are also more optimist, trying to adapt themselves, processing objective and symbolic data, establishing gregarious and flexible relationships. These characteristics had explained $37.9 \%$ of the variation in arterial blood pressure pre-dialysis. The MIPS discriminated different levels of compliance. The use of different indicators is important in the compliance assessment. The compliance levels observed agrees with the registered on literature.

Keywords: Health evaluation. Psychometrics. Patient compliance. Renal insufficiency chronic. 


\section{Introduç̃̃o}

Um dos problemas mais importantes para os profissionais de saúde atualmente é a aderência deficiente dos pacientes acerca dos regimes terapêuticos prescritos. As formas como os pacientes lidam com a enfermidade, com o regime terapêutico e com as necessidades de mudanças em seu estilo de vida representam pontos importantes para o curso da enfermidade, evolução do tratamento e repercussões em sua qualidade de vida (Martín Alfonso \& Grau Ábalo, 2005). No presente trabalho, define-se aderência terapêutica como o grau de concordância entre o comportamento de uma pessoa - tomar o medicamento, realizar dieta ou mudanças nos hábitos de vida - e as prescrições da equipe de saúde (Martín Alfonso, Bayarre Vea \& Grau Ábalo, 2008; Martín Alfonso \& Grau Ábalo, 2005; Organización Panamericana de Salud [OPAS/OMS], 2003, 2004).

Existem diversas estratégias e dificuldades na avaliação da aderência terapêutica, sobretudo em tratamento complexo ou para enfermidades crônicas. Delgado e Lima (2001) destacam duas estratégias metodológicas, os métodos diretos e objetivos - como o emprego de medições bioquímicas - e clínicas - como exames laboratoriais -, tomando-se a utilização de valores de referência padrão para a caracterização da aderência terapêutica. Outras estratégias são os métodos indiretos e subjetivos representados pelo autorrelato do paciente, o uso de entrevistas e também a contagem do medicamento.

Os métodos indiretos ou subjetivos estão sujeitos ao viés do entrevistador ou à imprecisão deliberada ou não do relato do paciente; há também a ressalva de que a contagem do medicamento dentro do consumo esperado não representa necessariamente que o paciente realmente ingeriu o medicamento. Já os métodos diretos e objetivos de medições bioquímicas são considerados estratégias diretas apenas por não serem mediados pela subjetividade de um profissional ou do paciente, mas também não representam uma relação direta e estreita entre aderência e resultados esperados. Observam-se dificuldades nesse tipo de medição, pois o paciente pode não aderir como esperado, mas obter a melhora em seu quadro clínico em função de outros fatores, além de também ser possível que mesmo que o paciente realize corretamente a terapêutica estabelecida não se observe os resultados desejados.
McDonald, Garg e Haynes (2008) destacam estratégias para avaliação da aderência estruturadas em torno de entrevistas com os pacientes, questionários e escalas, contagem dos medicamentos e medições bioquímicas. Em outro estudo, estes mesmos autores apontam três quesitos principais na avaliação da aderência terapêutica: o questionamento direto ao paciente, sua responsividade ao tratamento e o cumprimento das consultas agendadas (Haynes, McDonald \& Garg, 2008).

Mesmo diante desses apontamentos, o problema de uma avaliação consistente do processo de aderência persiste (Linck, Bielemann, Sousa \& Lange, 2008; Simpson Junior, 2008; Martin Alfonso \& Grau Abalo, 2005). Isso se reflete em uma grande amplitude nas estimativas acerca da aderência terapêutica. Delgado e Lima (2001) trazem dados que apontam a aderência oscilando entre 4 e $92 \%$, mas mais tipicamente variando dentro da faixa de 30 a $60 \%$. No mesmo sentido, Martin Alfonso e Grau Abalo (2005) indicam uma variação entre 33 e $82 \%$ dos pacientes que não seguem corretamente a prescrição medicamentosa. Kripalani, Yao e Haynes (2007) situam a não aderência entre 20 e $50 \%$ dos pacientes, e, especificamente no contexto da IRC, Leggat Junior (2005) menciona que a taxa de não aderência pode variar entre apenas $2 \%$ e $100 \%$ dos pacientes, dependendo da definição do termo aderência empregado e das estratégias de medição utilizados. Isso está de acordo com Delgado e Lima (2001), que explicam que essa grande variação é motivada pelas dificuldades metodológicas na avaliação da aderência.

Nesse cenário, o presente estudo busca avaliar aderência terapêutica no contexto do tratamento hemodialítico auxiliada pela avaliação psicológica como estratégia metodológica. A aderência terapêutica é parte do comportamento humano, implica na saúde e é expressão da responsabilidade dos indivíduos com os cuidados e manutenção da mesma. É o paciente quem executa ou não o consumo dos medicamentos e de outras indicações médicas, como seguir a dieta ou praticar exercícios. É ele quem decide em última instância como e quando tomará tais atitudes (Martín Alfonso, 2006).

Aspectos personológicos como motivação, crenças e cognições, estilos de personalidade, estratégias de enfrentamento são elementos que contribuem para essa tomada de decisões do paciente acerca de sua saúde. São elementos mediadores do indivíduo 
com o meio, as políticas públicas de saúde, campanhas educativas, o relacionamento com a equipe de saúde e com a própria família. Esses demais aspectos do seu meio ajudam a completar o quadro de relações do indivíduo com sua saúde, dando um contexto mais amplo e concreto onde deve ser inserido o conjunto formado pela personalidade e a saúde.

Este trabalho utiliza a teoria da personalidade de Theodore Millon (López, 2000; Millon \& Davis, 2004; Weiss, 2002) por meio do Inventário Millon de Estilos de Personalidade, para avaliar sua adequação como instrumento para verificação da aderência terapêutica em paciente em tratamento hemodialítico na região da Grande Natal (RN). A concepção de personalidade desenvolvida por Theodore Millon é marcada pelo seu aspecto integrador dentro da Psicologia ao articular coerentemente constructos desenvolvidos em diferentes escolas como o comportamentalismo, a psicodinâmica e a psicologia evolutiva. Ele considera a personalidade como

um padrão de funcionamento, uma forma intrínseca de agir, que resulta de uma matriz de variáveis determinadas pelo desenvolvimento biopsicológico. 0 desenvolvimento da personalidade recebe a influência dos fatores biológicos e dos fatores psicológicos, que passam a interagir como em uma espiral sem fim, onde cada giro desta se constrói sobre as interações prévias, criando, assim, novas bases para as próximas interações (Alchieri, Cervo \& Nuñez, 2005, p. 177).

A ideia de que fatores biológicos e psicológicos interagem em uma espiral sem fim traz a noção de fluidez e dinamicidade da personalidade normal, que se expressa de forma plástica e adaptativa às necessidades do meio.

Entre as exigências do dia-a-dia para o paciente em tratamento hemodialítico, encontra-se a necessidade de lidar eficazmente com as demandas impostas pelo tratamento, com as mudanças comportamentais em seu estilo de vida, com os choques com seu conjunto de crenças e valores, resultando na aderência ao tratamento renal. A Doença Renal Crônica (DRC) constitui-se em uma perda irreversível da função renal. Em sua fase mais avançada, chamada de Insuficiência Renal Crônica (IRC), os rins perdem sua capacidade de manter a homeostasia, filtrando impurezas resultantes do metabolismo e minerais não aproveitados pelo corpo, estimulando a produção de hormônios e regulando a pressão sanguínea (Milhoransa, Berthollo \& Comerlato, 2005; Romão Junior, 2004). A hemodiálise substitui parcialmente a função renal, onde a circulação e filtração do sangue são extracorpóreas. Atualmente é considerado o tratamento mais eficaz no enfrentamento da IRC e corresponde a 90,8\% dos atendimentos de terapia renal substitutiva (Milhoransa et al., 2005; Sesso et al., 2007).

\section{Materiais e métodos}

\section{Desenho do estudo}

Este foi um estudo analítico transversal que empregou técnicas para investigação de indicadores comportamentais e estratégias de investigação do processo de aderência ao tratamento hemodialítico.

\section{Participantes}

0 trabalho foi realizado com pacientes portadores de IRC em tratamento hemodialítico por, pelo menos, seis meses, entre os períodos de agosto de 2007 e janeiro de 2008, em dois centros clínicos de diálise na região da grande Natal (RN). Considerouse como critérios de inclusão para o paciente participar da investigação:

a) ser portador de insuficiência renal crônica e estar em tratamento hemodialítico regular há pelo menos seis meses;

b) ser maior de idade e possuir autonomia de decisão acerca de si mesmo;

c) realizar diálise regulamente nos centros clínicos escolhidos e não apenas como paciente em permanência temporária na clínica;

d) aceitar de modo livre e esclarecido participar deste estudo, com base nos pressupostos da Resolução n. 196/96 do Conselho Nacional de Saúde.

\section{Instrumentos}

Utilizaram-se como instrumentos, um protocolo de coleta de dados clínicos, elaborado a partir 
de revisão da literatura pertinente e do julgamento de especialistas em nefrologia e diálise, objetivando fornecer parâmetros clínico-laboratoriais para a avaliação da aderência ao tratamento hemodialítico. Desse modo, estabeleceu-se que as medições dos últimos seis meses do clearance de ureia (Kt/v), do percentual de redução da ureia (PRU), do produto cálcio-fósforo ( $\mathrm{Ca} \times \mathrm{P})$, do ganho de peso interdialítico (GPI) e da pressão arterial pré- e pós-diálise (PA pré/pós) seriam indicadores dos níveis de aderência do paciente, conforme os resultados estivessem dentro da faixa de valores de referência.

Somado a esse protocolo, foi utilizado o Inventário Millon de Estilos de Personalidade (IMEP) como estratégia para identificar aspectos psicológicos. O IMEP é composto por três dimensões (cognição, motivação e relacionamentos interpessoais) e vinte e quatro fatores condizentes a essas dimensões, distribuídos entre 180 itens (Alchieri, Nuñez, Cervo \& Hutz, 2008). Cada um desses itens representam uma afirmativa acerca do sujeito, que deverá julgar se ela é verdadeira, correspondendo a uma descrição correta de si, ou falsa, sendo imprópria para descrever seu comportamento, pensamentos ou sentimentos.

Adiante se realiza uma descrição de cada fator, situando-o em seus pares e dimensões (López, 2000):

a) Metas motivacionais

- abertura/preservação - avalia a tendência do sujeito de adotar uma postura otimista ou pessimista em relação aos eventos de sua vida;

- modificação/acomodação - intenção do sujeito em agir ativamente sobre o seu meio ou de se acomodar ao mesmo;

- individualismo/proteção - tendência do sujeito em considerar a si próprio em suas decisões ou em levar em conta as demais pessoas.

b) Modos cognitivos

- extroversão/introversão - medida em que o sujeito busca informação em seu meio exterior ou em seu meio interior;

- sensação/intuição - medida em que o sujeito busca informações objetivas, concretas e tangíveis ou procura informações subjetivas, simbólicas e especulativas;
- reflexão/afetividade - forma de processamento da informação a partir de esquemas lógicos e racionais ou a partir de esquemas subjetivos e afetivos;

- sistematização/inovação - forma de processamento da informação a partir de esquemas prévios de conhecimento ou a partir da produção de novas estruturas para a assimilação do conhecimento.

c) Relações interpessoais

- retraimento/comunicabilidade - padrão de conduta interpessoal baseado na indiferença e falta de emotividade ou baseado numa postura de gregarismo social, obtendo estimulação, excitação e atenção nesse meio;

- dúvida/segurança - padrão de dúvida versus segurança ou de autoafirmação nos relacionamentos sociais;

- discrepância/conformismo - estilo de relacionamento interpessoal marcado pela independência ou pelo cooperativismo;

- submetimento/controle - conduta representada pela submissão ao demais ou pelo domínio nas situações sociais;

- insatisfação/concordância: padrão de interação interpessoal passivo-agressivo ou receptivo e flexível.

É importante destacar que os fatores que compõem cada par não são excludentes entre si, mas complementares. Não há oposição no sentido psicométrico, mas no nível teórico, onde cada fator representa um extremo em seu par, formando polos (López, 2000).

Utilizou-se ainda um roteiro de entrevista semiestruturada a fim de investigar a autoavaliação do paciente acerca de seu processo de aderência ao tratamento hemodialítico.

\section{Procedimentos de coleta e análise dos dados}

Os procedimentos de coleta dos dados foram organizados em duas etapas. Em um primeiro momento realizou-se o levantamento dos dados clínicos e laboratoriais dos pacientes a partir de seus prontuários. Em uma segunda etapa procedeu-se à realização de entrevistas individuais com os pacientes participantes e aplicação do instrumento de 
avaliação psicológica. A análise dos dados foi realizada a partir de ferramentas estatísticas, empregando-se o teste Qui-Quadrado e o teste de Levene com nível de significância de 5\%.

\section{Resultados}

Foram avaliados 80 pacientes, $51,3 \%$ do sexo feminino e $48,8 \%$ do sexo masculino, com idade mínima de 19 anos e máxima de 78 anos, sendo a média igual a 43,4 anos e desvio padrão de 13,25 anos. Quanto ao tempo de diálise, observou-se um mínimo de seis meses de tratamento e um máximo de 22,07 anos ( $\pm 4,24$ anos). Entre os pacientes, 50\% são casados, $40 \%$ são solteiros e $10 \%$ são divorciados ou viúvos. Em relação à escolaridade, 17,5\% não eram alfabetizados, 43,6\% cursaram o Ensino Fundamental, 31,3\% possuem o Ensino Médio e $5,1 \%$ estão cursando $(\mathrm{n}=3)$ ou concluíram o Ensino Superior ( $\mathrm{n}=1)$. Acerca da renda, $43,8 \%$ dos pacientes entrevistados ganham até um salário mínimo proveniente de aposentadoria ou benefício saúde; e $85 \%$ dos pacientes possuem rendimentos abaixo de quatro salários mínimos. Assim, 15\% dos pacientes possuem renda entre quatro e dez salários mínimos.

0 protocolo de coleta de dados clínicos possibilitou destacar indicadores clínico-laboratoriais para níveis de aderência ao tratamento prescrito. A Tabela 1 mostra a distribuição percentual da aderência conforme cada critério avaliado. Observam-se amplas variações na percentagem de pacientes que conseguem alcançar o valor de referência para cada critério. Nesse sentido, apenas 26,2\% dos pacientes obtiveram percentual de redução da ureia (PRU) dentro do esperado. Por outro lado, 78,8\% dos pacientes apresentaram ganho de peso interdialítico (GPI) inferior a 2,5 kg. A média da avaliação sobre a aderência terapêutica ficou em $55,97 \%$ para a amostra investigada.

Acerca da autoavaliação do paciente sobre sua aderência, destaca-se primeiramente que $65 \%$ dos pacientes avaliaram positivamente sua aderência ao tratamento, contrapondo-se aos 35\% que indicaram uma aderência menos efetiva. Em segundo lugar, destaca-se a baixa significância indicada pelo teste Qui-Quadrado $(\mathrm{p}<0,05)$ entre a autoavaliação do paciente e a avaliação realizada com base nos critérios clínico-laboratoriais (Tabela 2). Apenas a avaliação com base no critério da pressão arterial pós-diálise apresenta uma associação significativa com a autoavaliação ( $p=0,029)$.

A Tabela 3 apresenta os dados acerca da avaliação da aderência ao tratamento segundo os critérios clínico-laboratoriais e dos aspectos avaliados pelo Inventário Millon de Estilos de Personalidade. A partir do kt/v observa-se que o modo cognitivo extroversão e o fator comunicabilidade - dentro da dimensão relações interpessoais - apresentam resultados bastante próximos entre os grupos de pacientes menos e mais aderentes; com escores levemente inferiores entre os pacientes menos aderentes. Contudo, os fatores afetividade, vacilação e conformismo apresentam valores claramente inferiores entre os sujeitos menos aderentes do que o observado entre os mais aderentes.

Ainda na Tabela 3, ao se identificar o PRU, observa-se que os fatores extroversão e comunicabilidade, pertencentes às dimensões modos cognitivos e relações interpessoais, respectivamente, apresentam um comportamento semelhante ao registrado junto ao kt/v: escores inferiores para os pacientes menos aderentes. Além desses itens, o fator firmeza - caracterizado pela autoafirmação nas relações interpessoais - se apresenta com escores bastante próximos entre os grupos de aderência, mas levemente superior entre os pacientes menos aderentes.

$\mathrm{O}$ produto cálcio vezes fósforo $(\mathrm{C} \times \mathrm{P})$, a pressão arterial pré-diálise (PA Pré) e pós-diálise (PA Pós) trazem uma relação significativa $(\mathrm{p}>0,05) \mathrm{com}$ um fator cada. Extroversão junto ao Ca×P e firme$z a$ a partir da pressão arterial pré-diálise reaparecem como já havia acontecido com o kt/v e o PRU.

Tabela 1 - Percentagem de pacientes mais e menos aderentes conforme os critérios clínico-laboratoriais

\begin{tabular}{lcccccccc}
\hline & kt/v & PRU & Ca×P & GPI & PA Pré & PA Pós & Autoavaliação & Média \\
\hline Mais aderentes & 51,2 & 26,2 & 67,5 & 78,8 & 53,15 & 72,5 & 65 & $55,97 \pm 18,37$ \\
Menos aderentes & 48,8 & 73,8 & 32,5 & 21,2 & 46,85 & 27,5 & 35 & $44,02 \pm 18,37$ \\
\hline
\end{tabular}

Fonte: Dados da pesquisa. 
Contudo, o fator firmeza observado agora traz um resultado inverso com um escore claramente inferior para o grupo de pacientes menos aderentes. Já o fator extroversão apresenta o mesmo comportamento anterior. 0 critério clínico pressão arterial pós-diálise coloca um fator novo, o modo cognitivo intuição, que apresenta um escore superior para os menos aderentes do que para os mais aderentes.

A autoavaliação dos pacientes acerca de sua aderência ao tratamento demonstrou uma relação significativa $(\mathrm{p}<0,05)$ com os fatores do IMEP apenas a partir da separação por sexo (Tabela 4). Nessa condição, observa-se o fator acomodação como

\section{Tabela 2 - Associação entre a autoavaliação do paciente e os demais critérios acerca da aderência ao tratamento}

\begin{tabular}{ll}
\hline Critérios de aderência & $\mathrm{p}^{*}$ \\
\hline $\mathrm{kt} / \mathrm{v}$ & 0,981 \\
$\mathrm{PRU}$ & 0,156 \\
$\mathrm{Ca} \times \mathrm{P}$ & 0,96 \\
$\mathrm{GPI}$ & 0,977 \\
PA Pré & 0,368 \\
PA Pós & 0,029 \\
\hline
\end{tabular}

Legenda: * = Teste Qui-Quadrado.

Fonte: Dados da pesquisa. sendo significativo $(\mathrm{p}=0,033)$ entre as pacientes do sexo feminino e o fator proteção para os pacientes masculinos $(p=0,025)$. Ambos os fatores representam aspectos motivacionais.

Por último, a Tabela 5 destaca os resultados da regressão linear dos critérios de aderência utilizados em relação aos fatores do Inventário Millon de Estilos de Personalidade.

A Tabela 5, a partir da maior proporção da variabilidade entre os critérios de aderência ao tratamento, observa-se que $37,9 \%$ da variação da pressão arterial pré-diálise é explicada pelo conjunto de fatores do Inventário Millon de Estilos de Personalidade. Logo em seguida, esse conjunto de fatores explica entre $33,2 \%$ e $23,3 \%$ a variabilidade dos demais critérios de aderência.

\section{Discussão}

0 resultado médio de todos os critérios descritos na Tabela 1 aponta para uma aderência em torno de $55,97 \%(\mathrm{dp}=18,37)$. Esse resultado está de acordo com o registrado pela literatura internacional, pois, ao lado das estimativas mínimas e máximas acerca da aderência, também se observam estimativas sobre a aderência média para diversas situações. Para Martín Alfonso e Grau Ábalo (2005), a aderência média às enfermidades agudas situa-se em torno de

Tabela 3 - Avaliação da aderência ao tratamento segundo os critérios clínico-laboratoriais para o IMEP

\begin{tabular}{lllrrrr}
\hline $\begin{array}{l}\text { Critérios clínico- } \\
\text { laboratoriais }\end{array}$ & Dimensões & Fatores & F & p* & Menos aderência & Mais aderência \\
\hline & Modos cognitivos & Extroversão & 12,046 & 0,001 & $27,32 \pm 7,68$ & $27,81 \pm 5,40$ \\
& & Afetividade & 4,506 & 0,037 & $33,32 \pm 7,34$ & $35,30 \pm 5,62$ \\
$/ \mathrm{v}$ & Comunicabilidade & 4,171 & 0,045 & $40,08 \pm 10,62$ & $40,16 \pm 8,29$ \\
& Relações & Vacilação & 7,86 & 0,006 & $21,84 \pm 10,49$ & $25,40 \pm 7,73$ \\
& Interpessoais & Conformismo & 13,188 & 0,001 & $48,68 \pm 8,75$ & $50,77 \pm 4,54$ \\
& Modos cognitivos & Extroversão & 8,319 & 0,005 & $27,05 \pm 7,08$ & $29,00 \pm 4,58$ \\
PRU & Relações & Comunicabilidade & 8,427 & 0,005 & $39,88 \pm 10,35$ & $40,77 \pm 6,30$ \\
& Interpessoais & Firmeza & 5,682 & 0,02 & $34,84 \pm 8,73$ & $34,77 \pm 5,09$ \\
Ca $\times$ P & Modos cognitivos & Extroversão & 10,802 & 0,002 & $27,15 \pm 8,14$ & $27,80 \pm 5,65$ \\
PA Pré & Relações & Firmeza & 4,691 & 0,033 & $33,86 \pm 8,74$ & $36,11 \pm 6,38$ \\
\hline PA Pós & Interpessoais & Intuição & 4,622 & 0,035 & $26,47 \pm 7,14$ & $26,18 \pm 8,79$ \\
\hline
\end{tabular}

Legenda: ${ }^{*}=\mathrm{p}<0,05$ no Teste de Levene.

Fonte: Dados da pesquisa. 
Tabela 4 - Avaliação da aderência ao tratamento segundo a autoavaliação dos pacientes para o IMEP

\begin{tabular}{lllcccc}
\hline Sexo & Dimensões & Fatores & F & p $^{*}$ & Menos Aderente & Mais Aderente \\
\hline Feminino & Metas Motivacionais & Acomodação & 4,927 & 0,033 & $24,00 \pm 6,63$ & $28,92 \pm 6,29$ \\
Masculino & Metas Motivacionais & Proteção & 5,416 & 0,025 & $32,00 \pm 5,97$ & $35,68 \pm 4,16$ \\
\hline
\end{tabular}

Legenda: ${ }^{*}=\mathrm{p}<0,05$ no Teste de Levene.

Fonte: Dados da pesquisa.

Tabela 5 - Regressão linear dos critérios de aderência ao tratamento com os fatores do IMEP

\begin{tabular}{lcccc}
\hline Critérios de Aderência & $\mathbf{R}$ & R quadrado & R quadrado corrigido & Erro típico de estimação \\
\hline PA Pré &, $615(\mathrm{a})$ & 0,379 & 0,124 & 0,4657 \\
GPI &, $576(\mathrm{a})$ & 0,332 & 0,057 & 0,39971 \\
PA Pós &, $550(\mathrm{a})$ & 0,302 & 0,016 & 0,48335 \\
Autoavaliação &, $533(\mathrm{a})$ & 0,285 & $-0,009$ & 0,48218 \\
kt/v &, $524(\mathrm{a})$ & 0,275 & $-0,023$ & 0,50756 \\
PRU &, $521(\mathrm{a})$ & 0,272 & $-0,027$ & 0,45545 \\
Ca×P &, $482(\mathrm{a})$ & 0,233 & $-0,083$ & 0,49039 \\
\hline
\end{tabular}

Legenda: (a) = variável dependente: (Constante), Concordância, Extroversão, Sensação, Afetividade, Acomodação, Reflexão, Controle, Sistematização, Retraimento, Abertura, Proteção, Firmeza, Discrepância, Conformismo, Insatisfação, Introversão, Individualismo, Preservação, Intuição, Modificação, Comunicabilidade, Inovação, Vacilação.

Fonte: Dados da pesquisa.

$80 \%$, enquanto nas enfermidades crônicas aproxima-se de 55\%. A OPAS/OMS (2003) afirma que, em média, $50 \%$ dos pacientes crônicos cumprem adequadamente o tratamento; Delgado e Lima (2001) trazem uma média de $40 \%$.

Entretanto, a autoavaliação dos pacientes acerca de sua aderência ao tratamento (Tabela 1) mostra que $65 \%$ dos pacientes entrevistados consideram-se aderentes. Esse valor se situa acima da média descrita na literatura anteriormente citada, e se aproxima das estimativas mais positivas sobre aderência ao tratamento. Tal dado é positivo, pois Resende, Santos, Souza e Marques (2007) relatam que uma avaliação positiva do paciente renal acerca de sua capacidade de lidar satisfatoriamente com as demandas do tratamento hemodialítico está diretamente relacionada com uma melhor aderência ao tratamento. No mesmo sentido, Curtin et al. (2008), em um estudo com 174 pacientes renais crônicos, apontam que essa autoavaliação positiva associa-se a um maior comprometimento do paciente com o tratamento, especialmente na aderência medicamentosa.
A avaliação psicológica realizada com os pacientes pode identificar características distintas entre os sujeitos mais aderentes e menos aderentes com perfis próprios dentro da amostra pesquisada. Seguindo uma linha de pesquisa semelhante, Solano, Casullo e Pérez (2004) conduziram um estudo no qual foram avaliados pelo IMEP 130 pacientes com transtornos orgânicos. Tomou-se como grupo controle um subgrupo da amostra de padronização do IMEP para a Argentina. Esses autores apontaram que foi possível diferenciar os pacientes com transtornos orgânicos da população geral, destacando-se o fator abertura entre as Metas Motivacionais. Esse dado é semelhante ao encontrado aqui para os pacientes mais aderentes, fazendo-se a ressalva de que enquanto os autores comparam grupos de pacientes com transtornos orgânicos com a população geral, no presente trabalho a comparação é feita com pacientes renais crônicos entre si, conforme o grau de aderência ao tratamento.

Desse modo e a partir dos aspectos motivacionais, pode-se afirmar que os pacientes mais aderentes são mais otimistas, sendo que os homens tendem 
a agir mais sobre seu meio, enquanto as mulheres buscam se acomodar mais à situação. Dessas características destaca-se a postura mais otimista para os sexos masculino e feminino e, particularmente, a motivação para a ação no homem, podendo remeter ao papel social do homem como provedor. A motivação para a acomodação na mulher é entendida como uma busca por aceitação e adaptação às novas circunstâncias vivenciadas pelo sujeito. Thomas e Alchieri (2005) encontraram dados semelhantes e acrescentando que os pacientes por eles avaliados como não aderentes obtiveram uma pontuação maior no Inventário Beck de Depressão. Almeida e Meleiro (2000) apontam que a depressão é a complicação psiquiátrica mais comum entre os pacientes renais crônicos, sendo fortemente associada à baixa aderência ao tratamento, perda de qualidade de vida e menor sobrevida.

Acerca dos modos cognitivos, os homens mais aderentes oscilam mais entre as fontes internas e externas de informação, buscando um pouco mais os dados subjetivos e especulativos de seu meio. Já as mulheres mais aderentes baseiam-se mais nos dados externos, mas oscilando entre o concreto e o subjetivo. Ambos também oscilam entre as formas de processamento desses dados, entre o modo de pensar mais lógico e racional e a forma de pensar orientada pela afetividade e subjetividade. Apenas entre as mulheres observou-se uma característica a mais da sistematização desse pensamento, tentando enquadrá-lo em esquemas prévios de conhecimento. No trabalho de Solano et al. (2004), destaca-se na dimensão modos cognitivos o predomínio dos fatores introversão, sensação e afetividade entre os pacientes com transtornos orgânicos. Esses dados implicam, dentro do aspecto cognitivo, num sujeito voltado para seu meio interno e para dados concretos, processando-os mediante parâmetros subjetivos e afetivos. O observado nesse estudo aponta para os pacientes mais aderentes, a meio caminho do que os citados autores encontraram para os transtornos orgânicos, enquanto os pacientes menos aderentes estão mais próximos desse perfil.

Os pacientes mais aderentes estão marcados por um estilo interpessoal gregário, mais seguros de si, percebendo-se como cooperativos e submissos e sendo mais receptivos e flexíveis às demandas sociais. Essas características de relacionamento interpessoais se conformam com um relacionamento mais saudável, fortalecendo a rede de apoio social e minimizando conflitos. Diversos estudos apontam para a importância do suporte social no processo de aderência terapêutica (DiMatteo, 2004; OPAS/ OMS, 2004). Coloca-se então a consonância entre a ocorrência de apoio social e essas características de comportamento entre os pacientes renais mais aderentes. Os sujeitos menos aderentes foram também os que obtiveram menores escores nos fatores da dimensão interpessoal. Solano et al. (2004) ressaltam que os pacientes orgânicos avaliados por eles apresentaram um escore maior no fator retraimento do que a população geral. Esse fator representa o polo oposto ao fator comunicabilidade e significa um padrão de relacionamento mais isolacionista e pouco emotivo nas interações sociais (Casullo, 2000).

Essas características que compõem o perfil descrito do paciente acerca de sua aderência conseguem explicar até $37,9 \%$ da aderência definida segundo um dos indicadores clínicos-laboratoriais. Esse valor é um pouco superior ao encontrado por Solano et al. (2004), que obtiveram uma função discriminante capaz de explicar $36 \%$ da variação de sua amostra.

\section{Conclusões}

O uso de estratégias distintas e paralelas na avaliação da aderência ao tratamento hemodialítico mostra-se como uma alternativa de se abarcar de forma mais global o fenômeno da aderência terapêutica. Entre essas estratégias há as contribuições da avaliação psicológica como uma útil ferramenta nessa investigação. Fernández-Ballesteros (1996) destaca a importância da avaliação psicológica no campo da saúde como uma estratégia auxiliar na promoção da saúde e prevenção de enfermidades, por meio da identificação de variáveis comportamentais relevantes. Nesse sentido, o processo de investigação psicológica da aderência terapêutica objetivaria complementar outras estratégias de avaliação por meio da identificação de aspectos comportamentais, cognitivos e motivacionais pertinentes. Dentro dessa perspectiva, a avaliação psicológica traria subsídios para uma investigação prognóstica da aderência do paciente, apontando pontos a serem trabalhados e avaliando a evolução do paciente, a eficácia das intervenções realizadas junto ao mesmo e complementando outras avaliações da equipe de saúde. 
O Inventário Millon de Estilos de Personalidade foi capaz de identificar características comportamentais - entre aspectos motivacionais, cognitivos e interpessoais - distintas em meio aos pacientes mais e menos aderentes. Solano et al. (2004, p. 135) afirmam que "los pacientes que sufren de alguna dolencia médica obtienen puntuaciones diferenciales en el MIPS". Esses autores se remetem ao uso do IMEP como instrumento auxiliar na avaliação de estilos de comportamentos pertinentes à avaliação da saúde do paciente. Desse modo, relacionam o uso desse instrumento de avaliação psicológica com outras estratégias de avaliação médica ou dos demais profissionais de saúde.

São necessários ainda outros estudos a fim de validar as características aqui descritas acerca do comportamento do paciente renal mais aderente ao tratamento e verificar os resultados obtidos em uma avaliação longitudinal dos pacientes, ampliando os resultados descritos. O seguimento dessas investigações em estudos posteriores possibilitaria uma discussão mais ampla desses resultados, além da discussão de novas hipóteses pertinentes como a possibilidade e utilidade da avaliação psicológica na complementação de dados prognósticos sobre a aderência terapêutica.

\section{Referências}

Alchieri, A. C., Cervo, C. S., \& Núñez, J. C. (2005). Avaliação de estilos de personalidade segundo a proposta de Theodore Millon. Psico, 36(2), 175-179.

Alchieri, J. C., Núñez, J. C., Cervo, C. S., \& Hutz, C. S. (2008). Características de validade convergente e divergente de instrumentos de avaliação da personalidade com o inventário de estilos de personalidade de Millon. Aletheia, 28, 119-134.

Alchieri, J. C. (2007). Avaliação da personalidade através do modelo teórico de estilos de personalidade de Millon In J. C. Alchieri (Org.) Avaliação Psicológica: Perspectivas e contextos (pp. 195-200). São Paulo: Vetor Editora Psicopedagógica.

Almeida, A. M., \& Meleiro, A. M. A. S. (2000). Depressão e insuficiência renal crônica: Uma revisão. Jornal Brasileiro de Nefrologia, 22(1), 192-200.
Casullo, M. M. (2000). Los estilos de personalidad: Una perspectiva iberoamericana. Madrid: Mino y Dávila. Mid:10966158.

Curtin, R. B., Walters, B. A. J., Schatell, D., Pennell, P., Wise, M., \& Klicko, K. (2008). Self-efficacy and self-management behaviors in patients with chronic kidney disease. Advances in Chronic Kidney Disease, 15(2), 191-205. doi:10.1053/j.ackd.2008.01.006.

Delgado, A. B., \& Lima, M. L. (2001). Contributo para a validação concorrente de uma medida de adesão aos tratamentos. Psicologia, Saúde e Doenças, 2(2), 81-100.

DiMatteo, M. R. (2004). Social support and patient adherence to medical treatment: A meta-analysis. Health Psychology, 23(2), 207-218. doi:10.1037/0278-6133.23.2.207.

Fernández-Ballesteros, R. (1996). Evaluación en psicología de la salud: Algunos problemas metodológicos. In M. M. Casullo (Org.) Evaluación psicológica en el campo de la salud (pp. 39-89). Barcelona: Paidós.

Haynes, R. B., McDonald, H. P., \& Garg, A. X. (2008). Helping patients follow prescribed treatment: Clinical applications. JAMA, 288(22), 2880-2883. doi:10.1001/ jama.288.22.2880.

Leggat Junior, J. E. (2005). Adherence with dialysis: A focus on mortality risk. Seminars in Dialysis, 18(2), 137-141. doi:10.1111/j.1525-139X.2005.18212.x.

Linck, C. L., Bielemann, V. L. M., Sousa, A. S., \& Lange, C. (2008). The chronic patient in face of falling ill and the treatment compliance. Acta Paulista de Enfermagem, 21(2), 317-322. doi:10.1590/ S0103-21002008000200014.

López, M. P. S. (2000). El MIPS (Millon Indexo f Personality Styles), como medida de los estilos de personalidad. In M. P. S. López \& M. M. Casullo (Org.). Estilos de personalidad: Una perspectiva iberoamericana (p. 3544). Madrid: Miño y Dávila. PMid:10654578.

Kripalani, S., Yao, X., \& Haynes, R. B. (2007). Interventions to enhance medication adherence in chronic medical conditions. Archives of Internal Medicine, 167, 540550. doi:10.1001/archinte.167.6.540. 
Martín Alfonso, L., \& Grau Ábalo, J. (2005). La adherencia terapéutica como un problema de la psicología de la salud. In E. H. Meléndez \& Ábalo J. G. (Org.). Psicología de la salud: Fundamentos y aplicaciones. (pp. 567589). Guadalajara: Universidad de Guadalajara.

Martín Alfonso, L. (2004). Acerca del concepto de adherencia terapéutica. Revista Cubana de Salud Pública, 30(4).

Martín Alfonso, L. (2006). Repercusiones para la salud pública de la adherencia terapéutica deficiente. Revista Cubana de Salud Pública, 32(3).

Martín Alfonso, L., Bayarre Vea, H., \& Grau Ábalo, J. A. (2008). Validation of MBG Questionnaire (MartinBayarre-Grau) for the evaluation of therapy adherence in blood hypertension patients. Revista Cubana de Salud Pública, 34(1).

McDonald, H. P., Garg, A. X., \& Haynes, B. H. (2008). Interventions to enhance patient adherence to medication prescriptions: Scientific Review. JAMA, 288(22), 2868-2879. doi:10.1001/jama.288.22.2868.

Millon, T., \& Davis, R. (2004). Trastornos de la personalidad en la vida moderna. Barcelona: Masson.

Organización Panamericana de Salud [OPAS/OMS], (2004). Adherencia a los tratamientos a largo plazo: Pruebas para la acción. Ginebra: OMS.

Organización Panamericana de Salud [OPAS/OMS], (2003). Currents topics: Poor adherence to long-term treatment of chronic diseases is a worldwide problem. Revista Panamericana de Salud Pública. 2003, 14(3), 218-221. doi:10.1590/S1020-49892003000800013.
Resende, M. C., Santos, F. A., Souza, M. M., \& Marques, T. P. (2007). Atendimento psicológico a pacientes com insuficiência renal crônica: Em busca de ajustamento psicológico. Psicologia Clínica, 19(2) 87-99. doi:10.1590/S0103-56652007000200007.

Romão Júnior, J. E. (2004). Doença renal crônica: Definição, epidemiologia e classificação. Jornal Brasileiro de Nefrologia, 26(3), 1-3.

Sesso, R., Lopes, A. A., Thomé, F. S., Bevilacqua, J. L., Romão Júnior, J. E., \& Lugon, J. (2007). Resultados do censo de diálise da SBN, 2007. Jornal Brasileiro de Nefrologia, 29(4), 197-202.

Simpson Junior, R. J. (2008). Challenges for improving medication adherence. JAMA, 296(21), 2614-2616. doi:10.1001/jama.296.21.jed60074.

Solano, A. C., Casullo, M. M., \& Pérez, M. A. (2004). Aplicaciones del MIPS en los ámbitos laboral, educativo y médico. Buenos Aires: Paidós.

Thomas, C. V., \& Alchieri, J. C. (2005). Qualidade de vida, depressão e características de personalidade em pacientes submetidos à Hemodiálise. Avaliação Psicológica, 4(1), 57-64.

Weiss, L. G. (2002). Essentials of MIPS assessment. In S. Strack (Org.) Essentials of Million Inventories Assessment. (pp. 224-268). New York: John Wiley \& Sons, Inc. 